\title{
Proceedings of the 14th Annual Meeting of the Society for Pediatric Dermatology Quebec City, Quebec, Canada June 22-24, 1989
}

\author{
Sharon S. Raimer, M.D., ${ }^{*}$ Neil S. Prose, M.D., $\dagger$ Adelaide A. Hebert, M.D., $\ddagger$ and \\ James E. Rasmussen, M.D. $\S$
}

*University of Texas Medical Branch at Galveston, †State University of New York Health Science Center at Buffalo, $¥$ University of Texas Health Science Center at Houston, and §University of Michigan, Ann Arbor, Michigan

The fourteenth annual meeting of the Society for Pediatric Dermatology was held in Quebec City, Quebec, Canada and hosted by Drs. Bernice Krafchik, Lionel Boxall, and the dermatology staff of L'Hôtel-Dieu de Quebec, particularly Drs. Raymond Lessard and Richard Cloutier. The fifth annual Sidney Hurwitz Lecture was presented by Professor Ruggero Caputo of the University of Milan. He discussed the histiocytoses, which he classified by their clinical, histopathological, ultrastructural, and immunohistochemical characteristics.

Histiocytosis $X$ is a proliferation of unknown cause of a distinct histiocytic cell type containing Langerhans' granules in the cytoplasm and staining positively for $\mathrm{S}-100$ and $\mathrm{OK}-\mathrm{T} 6$. The disease can be divided into four clinical subtypes: Letterer-Siwe disease, Hand-Schüller-Christian disease, eosinophilic granuloma, and congenital self-healing reticulohistiocytosis. Letterer-Siwe is the acute disseminated form of histiocytosis $\mathrm{X}$. Cutaneous lesions are present in approximately $80 \%$ of cases, particularly on the trunk and scalp, and these lesions are frequently the presenting sign of the disease. Lesions may present as papules (often with scaling, purpura, or crusts), pustules, petechiae, and purpuric striae of the nail beds.

Hand-Schüller-Christian disease, seen most commonly in children 2 to 6 years of age, is characterized by bony defects, diabetes insipidus, exophthalmos, and skin lesions (present in $30 \%$ to $50 \%$ of patients). Skin lesions are frequently similar to those seen in Letterer-Siwe disease but occasionally also involve mucous membranes.

Skin lesions are rare in eosinophilic granuloma, the benign localized form of the disease. When present, they may involve the periorificial region and mucous membranes. The lesions of congenital self-healing reticulohistiocytosis appear at birth or shortly thereafter as large reddish brown nodules which generally begin to involute after approximately 1 month.

Approximately $50 \%$ of the histiocytes in all four clinical forms of histiocytosis $\mathrm{X}$ contain Langerhans' granules. These granules differ from those found in Langerhans' cells in that they may be longer, are frequently attached to plasma membranes, may appear clumped, and may be present in nuclei as a result of abnormal mitosis.

The non-X histiocytoses lack Langerhans' granules and include juvenile xanthogranuloma, generalized eruptive histiocytoma, benign cephalic histiocytosis, sinus histiocytosis with massive lymphadenopathy, xanthoma disseminatum, papular xanthoma, and reticulohistiocytosis.

Address correspondence to James E. Rasmussen, M.D., Department of Dermatology, University of Michigan Medical Center, 1910 Taubman Health Care Center, Ann Arbor, MI 48109 0314. 
Juvenile xanthogranuloma in the small nodular (papular) form may present with 2 to $5 \mathrm{~mm}$ irregularly scattered papules occasionally associated with café au lait spots. In the less frequent large nodular form, which may involve mucous membranes and occasionally internal organs, it may present with one or a few 10 to $20 \mathrm{~mm}$ nodules. All lesions resolve within 3 to 6 years. The most common extracutaneous manifestation of the small nodular form is ocular involvement, which may precede or follow cutaneous involvement. Only six cases of generalized eruptive histiocytoma, a condition in which hundreds of 3 to $10 \mathrm{~mm}$ reddish to bluish papules appear in successive crops, have been reported in children. No visceral lesions have been described. The condition eventually resolves spontaneously.

Benign cephalic histiocytosis develops during the first 3 years of life. Papular or maculopapular 2 to 3 $\mathrm{mm}$ yellow-brown lesions develop principally in the head and neck region, particularly on the posterior side, and regress spontaneously during childhood without scarring. Mucous membranes and viscera are uninvolved. Approximately 20 cases have been described.

Sinus histiocytosis is associated with massive lymphadenopathy, fever, anemia, leukocytosis, elevated erythrocyte sedimentation rate, and polyclonal hypergammaglobulinemia. Approximately $10 \%$ of patients develop red-brown or yellow-brown papulonodular lesions without any particular distribution. Spontaneous resolution generally occurs.

Xanthoma disseminatum, a benign histiocytosis very rarely seen in children, presents with redbrown papules involving the face, trunk, and proximal extremities. The papules tend to become confluent on flexural surfaces and in the perioral region. The eyes and mucous membranes are generally involved and the disease is associated with diabetes insipidus. Both the skin lesions and the diabetes insipidus resolve after several years.

Dr. Caputo has recently described papular xanthoma (previously reported only in adults) in two children. Yellowish papulonodular lesions without visceral involvement are clinically very similar to juvenile xanthogranuloma; histology is necessary for differentiation. Spontaneous resolution has been observed only in children.

Reticulohistiocytosis is a form of histiocytosis very rarely seen in children. A purely cutaneous solitary papulonodular form involving mainly the head and neck, and a diffuse widespread form not associated with arthritis may occur. Only two cases of multicentric reticulohistiocytosis associated with polyarthritis have been reported in children. The skin lesions associated with the disease are translucent yellow or yellow-brown papulonodules ranging in size from a few millimeters to two centimeters that occur most commonly on the hands and occasionally on the knees and face. Nasal and pharyngeal mucosal lesions may occur in approximately $50 \%$ of cases.

The next presentation was given by William Macrae, M.D., of the University of Toronto, who discussed diseases in which changes occur in both the skin and eye. Fifty percent of childhood blindness is due to inherited conditions and ten percent of visually-impaired children have oculocutaneous albinism as the basis of impairment. Albinism has several different genetic varieties: recessively inherited tyrosinase-positive or tyrosinase-negative oculocutaneous albinism affecting hair, skin, and eyes; or ocular albinism affecting eyes only, classically $\mathrm{X}$-linked in inheritance, but occasionally inherited as an autosomal recessive condition. Albinos demonstrate visual loss, photosensitivity, and nystagmus. The visual loss is due to central retinal hypoplasia, resulting in reduced central vision but normal visual fields. Optic fibers from the temporal retina to the ipsilateral geniculate nucleus are misrouted so that binocular vision is severely impaired. Tyrosinase-negative individuals generally have greater visual impairment. Dr. Macrae stressed that the hair bulb incubation test for the presence of tyrosinase is not reliable under 1 to 2 years of age.

Lisch nodules may be helpful in the diagnosis of neurofibromatosis. They are elevated, smooth, translucent lesions that are present bilaterally on the iris in $90 \%$ of neurofibromatosis patients over 6 years of age.

Infants with Sturge-Weber syndrome should be examined for the presence of congenital glaucoma due to choroidal hemangioma, and patients with a nevus of Ota should be followed closely because of an approximate $4 \%$ incidence of melanoma in these nevi.

Patients with atopic dermatitis frequently have an associated keratoconjunctivitis, which can be treated with cold compresses, antihistamines and the judicious use of topical steroids. Eight percent of atopics develop cataracts.

Thirty-five percent of patients with StevensJohnson syndrome have permanent sequelae because of scarring. Treatments during the acute phase of the disease include topical steroids, lubricants, and bandage contact lenses to maintain the integrity of the cornea. 
Herpes simplex of the cornea is the most common infectious cause of blindness. Keratitis is the most important manifestation; ulceration caused by the virus can be seen on fluorescein staining. Dr. Macrae prefers topical trifluridine (Viroptic) and oral acyclovir as treatment. Chronic conjunctivitis may be due to herpes simplex; steroid-containing eyedrops should be avoided in patients with chronic conjunctivitis unless herpes simplex infection has been ruled out.

Eye involvement may occur with Lyme disease. In stage $I$ of the disease, conjunctivitis may occur and, rarely, a panophthalmitis resulting in blindness. In stage III of the disease, keratitis and uveitis have been described, as well as cranial nerve palsy.

Large strawberry hemangiomas of the eyelid may cause loss of vision due to occlusive amblyopia, strabismic amblyopia, or compression of the globe. Intervention with such modalities as argon laser, excision, or intralesional steroids must be attempted without delay to prevent permanent visual loss.

Hans-Michael Dosch, M.D., from the University of Toronto presented his recent studies on the formation of the IgE molecule. The genome for immunoglobulins consists of approximately 200 kilobases beginning with those coding for IgM then, sequentially, $\operatorname{IgD}, \operatorname{lgG}, \operatorname{IgA}$, and lastly, IgE. It is presently accepted that individual $\mathrm{B}$ cells begin as IgM secretors then subsequently become $\operatorname{IgD}, \operatorname{IgG}$, and $\operatorname{IgA}$ secretors. Finally, a few cells reach the "end of the line" and become IgE secretors.

Dr. Dosch's laboratory noted that lymphocytes with IgE on their plasma membranes also had the IgM molecule. When IgM-secreting cells were isolated from atopics, all cells that made $\operatorname{IgE}$ were also found in this group of cells. He believes that approximately $90 \%$ of B lymphocytes progress from making IgM to making IgA or IgG, while approximately $10 \%$ continue to make IgM and also make $\operatorname{IgD}$ and $\operatorname{IgE}$. Further studies on the regulation of this system are needed.

Colin Ramsay, M.D., from the University of Toronto discussed porphyria in children. The porphyrias can be divided into erythropoietic and hepatic types. The former group includes erythropoietic porphyria (Gunter's disease), erythropoietic protoporphyria (EPP), and erythropoietic coproporphyria. Gunter's disease presents in early childhood with marked hypertrichosis, mutilating skin changes, splenomegaly, and a marked hemolytic anemia that is frequently the cause of death. All tissues will fluoresce under a Wood's light.

Erythropoietic protoporphyria is the most com- mon porphyria of childhood and features symptomatic mild photosensitization. When present, skin changes consist of thickening on the nose and dorsum of the hands with mild scarring of the cheeks. Red blood cells (RBC), which contain high levels of protoporphyrin, fluoresce brilliantly for 20 to 30 seconds under a fluorescent microscope. Plasma protoporphyrin levels are elevated due to loose binding of protoporphyrins to RBCs. Urine and stool porphyrins are normal. Iron deficiency anemia and lead intoxication also result in elevated RBC protoporphyrin levels, although such patients are not photosensitive because the protoporphyrins are tightly bound to RBCs. Iron deficiency anemia in association with a polymorphic light eruption could be misdiagnosed as EPP due to an increase in red cell porphyrins associated with photosensitivity. Cirrhosis of the liver occurs in a small number of patients with EPP.

The hepatic porphyrias are acute intermittent porphyria, variegate porphyria, hereditary coproporphyria, and porphyria cutanea tarda. Variegate porphyria (VP) and hereditary coproporphyria (HCP) may present with acute systemic symptoms identical to those of acute intermittent porphyria, with skin findings which are identical to those of porphyria cutanea tarda (PCT), or a combination of both. Investigation of porphyrin levels in VP demonstrates coproporphyrin levels greater than uroporphyrin in urine and protoporphyrin greater than coproporphyrin in stool. Porphyrin levels are normal in red blood cells. Hereditary coproporphyria also shows levels of coproporphyrin higher than uroporphyrin in urine, but stool shows much higher levels of coproporphyrin than protoporphyrin. During acute episodes of either disease, the urine may also contain aminolevulinic acid and porphobilinogen. Porphyria cutanea tarda, which is quite rare in childhood, should only be diagnosed when VP and HCP have been ruled out thoroughly. All available family members of patients with VP or HCP should be screened. Diagnosing asymptomatic patients allows education about inciting drugs, thus preventing serious acute episodes of these diseases.

Lynn From, M.D., University of Toronto, presented a discussion on nevi in children. She began by discussing her view that large congenital nevi should not be removed as a cancer-preventing measure. She noted that death from melanoma in children under 10 years of age is extremely rare in the United States and no such death has been reported in Ontario in the last $\mathbf{3 0}$ years. It is well recognized, however, that deaths do occur from melanoma in association with large congenital nevi. Some of 
these melanomas actually arise in the central nervous system, which frequently contains increased numbers of melanocytes in association with large congenital nevi. Because large congenital nevi usually extend deeply into subcutaneous tissue, it is frequently impossible to remove all the nevus cells. Deaths have occurred from melanomas arising under skin grafts after extensive surgery to remove the lesions.

It has been noted in recent years that patients with a diagnosis of melanoma in a large congenital nevus frequently survive long-term. Dr. From noted that these nevi may develop nodules that contain areas of necrosis and hemorrhage and numerous mitotic cells that may be very difficult to distinguish from melanoma. She feels that many of these are due to trauma and have been misdiagnosed in the past as melanoma. Approximately $60 \%$ of large congenital nevi have many mitotic figures in the deep dermis. She noted that worrisome nodules that are present at birth frequently regress. Spitz nevi also can occur within congenital nevi and may be misdiagnosed as melanoma. Parents or patients may desire removal of congenital nevi for cosmetic reasons, but there appears to be no urgency in the removal and one may not be preventing the occurrence of melanoma by such removal.

In discussing small congenital nevi, Dr. From noted that in a 2-year study in Ontario approximately $1.9 \%$ of melanomas had evidence of the classic histology of a congenital nevus associated with the melanoma. She manages congenital nevi by giving parents a low-power photograph of the nevus for positional orientation and a close-up so that parents can look for changes in shape or color. She removes any nevus that has changed.

The speaker mentioned that she had recently seen two cases in which malignant melanoma developed in nevus spilus-like lesions. The dark areas in this type of lesion frequently have the histologic changes of dysplastic nevi. Spindle cell nevi typically arise rather quickly during childhood. Histologic changes occur mostly in the epidermis. Numerous mitotic figures are frequently present, and the lesion may be misdiagnosed as a malignant melanoma. Spitz nevi, which typically have more dermal involvement than spindle cell nevi, occasionally may be very difficult to distinguish from melanoma histologically, and clinical judgment may be of greater value than histopathology in children.

In dysplastic nevi, the atypical cells are present principally at the edges of the lesion. Generally the entire nevus should be removed for biopsy, but if a smaller biopsy is done, it should be taken from the edge of the lesion. Recent studies suggest that the more pigmented nevi one has, dysplastic or not, the greater the chance of developing melanoma. The number of pigmented nevi may be related to the number of sunburns an individual has had.

The "Cases of the Year' session was moderated by Sam Weinberg, M.D. Susan Mallory, M.D., presented the first case, a 4-year-old white girl with dermatomyositis in whom marked hypertrichosis was the presenting clinical sign of the disease.

Amy Paller, M.D., discussed a 5-year-old girl who developed left leg pain and edema followed by vasculitic changes with palpable purpura and necrosis. Computerized tomographic scans demonstrated thrombosis in the iliac and femoral veins. Direct immunofluorescent studies of a skin biopsy showed granular deposits of $\operatorname{lgG}$, IgM, and C3 around dermal blood vessels. An extensive immunologic workup was essentially normal except for the presence of anticardiolipin antibodies.

Antiphospholipid antibodies, which include anticardiolipin antibodies, and lupus anticoagulant are frequently present in association with collagen vascular diseases, particularly systemic lupus erythematosus and juvenile rheumatoid arthritis, but occasionally are seen in normal individuals. In adults these antibodies may be associated with recurrent thrombotic episodes, fetal loss from placental infarcts, thrombocytic anemia, Coombs' and hemolytic anemia, livedo reticularis, vasculitis, and rarely migraine headaches and seizures. This syndrome has not been reported in children.

Maureen Rogers, M.D., described the case of a 4-month-old boy with a viral pneumonitis managed under a croup tent who developed vesicles over the sites of venipuncture. The vesicles rapidly enlarged into bullae. Histology was consistent with miliaria, showing hyperkeratosis, very marked dilated sweat ducts with extravasation of sweat into the dermis, and a mild inflammatory infiltrate. No typical miliaria rubra was present. Although the lesions resolved spontaneously, two small lesions recurred with a subsequent febrile illness. Ron Hansen, M.D., reported having seen a similar eruption in a terminally ill young infant.

Patricia Treadwell, M.D., presented an 8-yearold girl with a lesion on the helix of the ear. The patient's mother had a similar sore on the lower leg. Sporothrix schenkii was isolated from both patients. Sporotrichosis in more than one family member has been reported, but is a rare occurrence.

Daniel Hogan, M.D., discussed the case of a healthy 7-year-old girl who presented with a two week history of an enlarging nodule of the wrist that 
progressed to ulceration. Routine histology showed a granulomatous infiltrate with giant cells. Numerous acid-fast bacilli were demonstrated with special stains. A mycobacterial infection was diagnosed and the child was treated for 2 months with INH and Rifampin. Cultures subsequently grew Rhodococcus terrae, a partially acid-fast, fairly ubiquitous soil organism. Treatment was discontinued and the lesion healed spontaneously.

Andrea Dominey, M.D., presented an infant with a large congenital tumor of the head and neck, which proved to be a rhabdoid sarcoma on histologic examination. Cutaneous metastases to the thigh and back were present at birth. This rare tumor has a rapidly fatal course. The mean age of onset in children is 18 months and the usual primary location in this age group is the kidney. In adults, this sarcoma may present as a subcutaneous tumor of the extremities or the oral cavity. The histologic hallmark of a rhabdoid sarcoma is the presence of eosinophilic cytoplasmic inclusions that are PAS positive, diastase resistant, and stain immunohistochemically for vimentin.

Linda Rabinowitz, M.D., described two infants with capillary hemangiomas treated initially at 4 days and 8 days of age with the $585 \mathrm{nM}$ pulse dye laser. The lesions initially became gray in appearance, then purpuric, and faded over 1 to 2 weeks. The $585 \mathrm{nM}$ pulse is within the absorption spectrum of oxyhemoglobin. The energy absorbed by oxyhemoglobin presumably damages endothelial cells, resulting in vessel closure, generally without scarring.

Steven Resnick, M.D., reported a child who developed a Bechet's-like syndrome with high fevers, uveitis, retinal vasculitis, facial pustules, and an erythema nodosum-like eruption on the legs shortly after having a nickel-containing orthodontic appliance placed in her mouth. The appliance was removed and the child has responded to a slow taper of systemic steroids.

Anne Lucky, M.D., presented an infant with congenital ichthyosiform erythroderma who was hospitalized three times before 3 months of age for severe failure to thrive. Oxygen consumption studies done at 3 months of age indicated that the infant had an increased energy expenditure and that his caloric requirement was almost twice that of a normal infant. With adequate caloric intake the infant gained weight and the skin became less erythrodermic.

The last cases of the session were presented by Carl Frix, M.D. He reported several children who developed urticaria and a serum sickness-like erup- tion following their second exposure to the antibiotic cefaclor (Ceclor).

On Friday morning, 23 June 1989, a series of clinical cases was presented to the meeting participants at L'Hôtel-Dieu de Quebec, the oldest hospital in North America. This remarkable program, organized by Drs. Raymond Lessard and Richard Cloutier, included 20 patients from throughout Quebec and Ontario, and eight poster presentations. The discussion that followed the patient viewing was led by Dr. Cloutier.

Dr. Danielle Marcoux of Hôpital Ste-Justine in Montreal presented a 6-year-old boy who developed a large area of atrophy on the right buttock and three smaller areas on the posterior aspect of the leg. A biopsy of the lesion showed atrophy of the subcutis with a perivascular lymphohistiocytic infiltrate. Conference participants agreed with the suggested diagnosis of lipodystrophy centrifugalis, and most felt that the prognosis for spontaneous resolution was quite good.

Dr. Marcoux also presented an 8-year-old girl with erythrokeratoderma variabilis. The patient had widespread erythematous, hyperkeratotic plaques involving the trunk and extremities. Cutaneous involvement ended sharply at the base of the neck, and there was a bizarre, spiralled gyrate erythema superimposed upon the areas of hyperkeratosis. Treatment with intermittent courses of oral 13-cis retinoic acid and topical keratolytics has been well tolerated, but the skin disease remains severe and extensive.

A 12-year-old girl with severe lamellar ichthyosis who had collodion features at birth was presented by Dr. Bertrand Rodrigue of Hospital St-Sacrement, Quebec. He reported significant therapeutic success with $5 \%$ glycolic acid in water washable base. Dr. Bernice Krafchik reported that collodion babies respond to therapy with the visible light that is used in the treatment of hyperbilirubinemia.

Dr. L. J. Margesson of Kingston, Ontario, presented a 32-month-old male who was born as a harlequin fetus. Treatment with oral etretinate began at 3 days of life. The child has persistent, diffuse erythema and scaling and is still on etretinate therapy. Long-term survival of a harlequin fetus remains an extremely unusual phenomenon.

Dr. Bernice Krafchik's case was a 5-year-old boy with recurrent asymptomatic reddish papules with ulceration and crusting since age 4 months. Repeated biopsies of these skin lesions led to the diagnosis of lymphomatoid papulosis. The occurrence of this disorder is extremely rare during childhood, 
and histologic confirmation will often require serial biopsies.

Dr. Jacques Soucy presented a 6-month-old girl with a cavernous hemangioma involving the face and neck. Dr. Anne Lucky noted that magnetic resonance imaging may be useful in determining the degree of anatomical involvement in the neck region, and may indicate when steroid therapy is needed.

Dr. Julie Powell and Dr. A. Bolduc presented a 7-year-old boy with a progressively enlarging asymptomatic lesion on the left thigh since age 2 years. The lesion was an indurated, violaceous plaque with focal areas of epidermal atrophy. After exhaustive laboratory investigation, the discovery of budding spores in giant cells of the skin and lymph nodes suggested the diagnosis of North American blastomycosis. Dr. Ilona Frieden pointed out that granulomatous slack skin disease may have a similar clinical appearance and suggested that it be considered as an alternative diagnosis.

A 15-year-old girl with extensive porokeratosis of Mibelli was described by Dr. Richard Cloutier. The patient also had a thick area of hyperkeratosis involving the left heel that interfered with walking. The lesion has responded well to laser therapy.

Dr. Danielle Marcoux presented a 16-year-old girl for diagnosis of a progressive dermatosis that had been present since the first year of life. The patient had developed linear atrophic bands of the posterior lower extremities, with similar lesions on the breasts and in the axillae and inguinal areas. Conference participants favored the diagnosis of focal dermal hypoplasia. Several discussants pointed out that the biopsy of this disorder may be difficult to interpret if obtained from an early lesion. Histologic comparison with an area of normal skin may sometimes be helpful.

The Saturday session was moderated by Walter Tunnessen Jr., M.D., and opened with Dr. William Weston's "Cases in Search of a Diagnosis." The first case was that of a 36-week-old female infant with a diffuse, "woody" nonpitting induration of the skin, arthrogryposis, and hypoplastic lungs. Thick skin was noted by ultrasound done at 26 weeks for decreased fetal movement. Presenters Moise Levy, M.D., and Andrea Dominey, M.D., of Houston, Texas, described sclerematous induration of the skin at birth. At autopsy, the infant had "policeman's tip" positioning of the arms and fatty hypoplasia of the muscles. Biochemical studies were unremarkable and viral cultures were negative. In his differential diagnosis, Dr. Weston included 1) restrictive dermopathy (J Invest Dermatol 1987; 88:330-339); 2) Pena-Shokeir phenotype (J Pediatr 1974;85:373-375); and 3) sclerema from in-utero hypoxia (Am J Obstet Gynecol 1986;155:380-381). Dr. Andrew Margileth had seen similar cases that he felt resulted from congenital cytomegalovirus infection and congenital varicella. No conclusive diagnosis was reached, however.

The second case was from Dr. Weston's department in Denver. A 61/2-year-old boy had been in good health until January 1989, when he developed streptococcal pharyngitis and 2 weeks of hematuria. After the resolution of the hematuria, he developed recurrent episodes of fever up to $103^{\circ}$ Fahrenheit for 3-day intervals. He had an episode of urticarial rash on the distal extremities and was thought to have juvenile rheumatoid arthritis. Therapy with aspirin resulted in only slight improvement.

Dermatologic evaluation was sought when the child developed new, bullous skin lesions that began on the dorsal feet, spreading to other parts of the body. The bullae were often hemorrhagic. The child was on enteric coated aspirin when the eruption occurred. A skin biopsy showed a subepidermal split with no vasculitis. Direct and indirect immunofluorescence was negative on two occasions. The sedimentation rate, which had been $70 \mathrm{~mm} / \mathrm{hr}$, was $7 \mathrm{~mm} / \mathrm{hr}$ at the time the acute bullous eruption began. A normochromic anemia and a slightly elevated eosinophil count were reported. The antinuclear antibody titer was negative, and the antistreptolysin titer was not elevated. Dr. Lynn Roberts recommended that indirect immunofluorescence be done on sodium chloride split skin. The differential diagnosis included linear IgA dermatosis, bullous drug eruption, bullous pemphigoid, lupus erythematosus, and Henoch-Schonlein purpura. Therapy had included $500 \mathrm{mg}$ oral sulfapyridine twice daily for 12 days, which resulted in nausea, vomiting and diarrhea. Steroids had not been used in therapy.

The second speaker was Carol Redmond, M.D., from the Department of Obstetrics and Gynecology at the Hospital for Sick Children in Toronto, who discussed lichen sclerosus et atrophicus (LSA) in children. Dr. Redmond supports the view that LSA in girls is similar to the form seen in adult women in its symptomatology, appearance and histologic changes. The two forms differ, however, in their natural history. LSA may not be rare in children, and most often Caucasian females are afflicted with this disorder. The earliest recognized case occurred at 1 month of age with a peak incidence at 3 to 6 years. 
Symptoms may be intermittent, with episodes of vulvar pruritus lasting $I$ to 2 weeks. Anal fissures may develop and predispose to constipation. Clinically apparent genital lesions tend to be symmetric and can involve the vulva (primarily the labia majora), the clitoris, or the vestibule. The skin changes can include leukoderma, erythema, telangiectases, hemorrhagic bullae, and fissures. The alterations may wax and wane and, on rare occasions, predispose to gross contractures of the vaginal introitus. Kraurosis vulvae is an exceedingly rare sequelae of LSA in girls. Friedrich (Friedrich G, Jr. Vulvar Disease, 2nd ed. Philadelphia: WB Saunders, 1983) describes a phenomenon known as agglutination where there is loss of the labia minora due to adhesions from LSA.

The differential diagnosis of LSA presented by Redmond included 1) monilia (although the absence of glycogen in prepubertal girls makes this diagnosis unlikely), 2) nonspecific vulvovaginitis, 3) herpes vulvitis, 4) sexual abuse, 5) vitiligo, and 6) masturbation.

Does LSA spontaneously resolve in young girls? Dr. Redmond found that $50 \%$ to $60 \%$ of 72 girls studied previously got better symptomatically. The recovery period averaged 5 years with a range of 1 to 10 years. In data collected at the Hospital for Sick Children LSA Clinic in Toronto, 42 of 55 patients (76\%) got better symptomatically (i.e., still had itching, but less often) with an average recovery period of 3 years (range 0.2 to 7 years). Only $23 \%$ had resolution of their cutaneous changes. Relapses have occurred even after 4 years of being symptom free. Involution of prepubertal anogenital LSA occurred in two-thirds of cases before or around menarche. In Dr. Redmond's study, 75\% had symptomatic improvement unrelated to puberty and $60 \%$ were still symptomatic at puberty. No known predictive factors exist that determine which patients will have resolution of their LSA at menarche. Therapeutic modalities include topical steroids and topical hormonal agents. Topical steroids are the mainstay of therapy in girls with LSA and do seem to help control the itching.

In his second address, Professor Ruggero Caputo presented an overview of papular eruptions in children and briefly described the cutaneous manifestations that followed the 1976 tetrachlorodibenzodioxin (TCDD) spill in Seveso, Italy. The lichenoid eruptions encompass lichen planus, lichen nitidus, lichen striatus, papular acrodermatitis of childhood, papulovesicular acrolocated syndrome, and pityriasis lichenoides.

Lichen planus (LP) occurs rarely in infancy and childhood with less than $2 \%$ of all cases seen in patients under 20 years of age. If the LP is familial it may occur at a younger age. Although oral mucous membrane lesions are rare in childhood lichen planus, the nails may be involved ( 20 nail dystrophy).

Characterized by asymptomatic 2 to $3 \mathrm{~mm}$ papules, lichen nitidus is easily differentiated from LP based on clinical and histologic features. The hair, nails, face and oral mucous membranes are typically spared. Males are affected four times as often as females.

Greater than $50 \%$ of all cases of lichen striatus occur in children. Onset is usually between the ages of 2 and 15 years. More than one linear band may be seen distributed along the lines of Blaschko.

Papular acrodermatitis of childhood (PAC) was distinguished from papulovesicular acrolocated syndrome (PAS) (J Am Acad Dermatol 1982;6:862-866). In Europe, the hepatitis virus subtype ayw is the form most frequently associated with PAC, and children with HLA Bw 54 are significantly more likely to develop the disease. The oldest patient to develop PAC in Dr. Caputo's series was 12 years of age.

Pityriasis lichenoides was divided into three groups (Table 1) based on distribution and duration of the eruption. No relationship existed between the age of onset and the ultimate course of the disease. Similarly, no relationship has been recognized between topographical distribution and the severity of the disease. None of Dr. Caputo's cases went on to develop lymphomatoid papulosis.

Seveso, Italy was the site of a large TCDD spill on 10 July 1976. The chemical spread through the air to the surrounding 16 kilometers. Both acute and delayed onset of cutaneous reactions to the chemical were illustrated by Dr. Caputo. The lesions felt to be due to direct toxic exposure were noted within 24 to 48 hours. Erythema and edema of exposed areas of skin, vesiculobullous and necrotic papules of the palms and soles, and papulonodules on the arms and chest developed acutely. One to two months after the chemical spill, cases of chloracne occurred, believed due to both direct exposure of the skin to TCDD and ingestion of contaminated foods. Lesions of chloracne resolved slowly, leaving pitted scars.

TABLE 1. Pityriasis Lichenoides in Children

\begin{tabular}{|c|c|c|}
\hline Form & Location & $\begin{array}{l}\text { Duration } \\
\text { (months) }\end{array}$ \\
\hline $\begin{array}{l}\text { Diffuse } \\
\text { Central } \\
\text { Peripheral }\end{array}$ & $\begin{array}{l}\text { Face, trunk, limbs } \\
\text { Lacalized on trunk } \\
\text { Limbs, face }\end{array}$ & $\begin{array}{l}11.4 \\
17.3 \\
31.3\end{array}$ \\
\hline
\end{tabular}


Judah Folkman, M.D., introduced many emerging concepts with regard to angiogenesis (N Engl J Med 1989;320:1211-1212). He described the six angiogenic polypeptides now recognized: fibroblast growth factor, angiogenin, transforming growth factors alpha and beta, tumor necrosis factor alpha, and platelet-derived endothelial cell growth factor. Theoretically, if we could control angiogenesis, we could gain control of a number of diseases, such as hemangiomas and psoriasis. An alternative hypothesis is that port wine stains are not the result of excess angiogenesis, but arise due to a defect in smooth muscle. The ratio of endothelial cells to smooth muscle cells may serve as a reliable equation to determine the nature of vascular malformations. In port wine stains, the ratio of these elements is 396 endothelial cells to 42 smooth muscle cells $(9: 1)$. One of the most promising drugs that may have clinical implications in managing vascular abnormalities is interferon $\alpha$. This drug has shown promise in the treatment of pulmonary capillary hemangiomatosis (N Engl J Med 1989;320:1197$1200)$.

The Resident Award paper was presented by Glenn Kline, M.D., whose colleagues were Oscar L. Frick, M.D., Ph.D.; Edward L. Strem, M.D.; and Mary L. Williams, M.D. His research entailed a study of ten patients with atopic dermatitis who were treated with a slow-release form of ascorbic acid (Geriatric Pharmaceutical Corporation). All patients were between the ages of 3 and 21 years (average age, 8 years), six were male, four were female, and all had lgE levels in excess of $2000 \mathrm{IU} / \mathrm{ml}$. No adverse effects of the vitamin $C$ therapy were noted. Fewer courses of antibiotics were required by patients on the vitamin $\mathrm{C}$ during the 3-month treatment period as compared to controls.

Practical pointers on how to approach drug studies were offered by Dr. John Arlette. The use of multiple sites in performing drug studies is helpful in offsetting local variations. The disease being studied should be clearly defined in terms of clinical, biochemical and/or histologic findings. Ensuring compliance is often a major obstacle in clinical drug study trials. Compliance may be enhanced by frequent patient follow-up visits, giving additional attention to each patient, selecting patients on their ability to comply, and counting the pills prescribed at each visit. Errors in drug studies may arise as a function of the impact of environmental conditions on the disease, the patient's desire to perform, and intolerance to vehicles being studied.
The pharmacologic diagnosis of drug-induced skin disease was presented by Stephen Spielberg, M.D. Drugs may induce multisystem disease with fever and rash. In any given individual, a drug eruption may develop as an expression of a hypersensitivity reaction. These reactions may be related to the innate ability to acetylate a drug or to biochemical abnormalities. Currently, studies are underway to define the pharmacogenetic basis of drug reactions. The Pandix plate with fluorescent probes serves as a tool in those investigations.

Dr. Samuel Weinberg introduced each speaker in the final session, "Cases of the Year." Some of the cases are described below. Dr. Moise Levy described several cases of Naprosyn photosensitivity in children. Histologically, a subepidermal bulla was characteristic, as well as photodistributed increased skin fragility in some patients.

A child with manifestations of congenital leukemia within the first 3 days of life was detailed by Dr. William Bason. Dr. Julie Prendiville's case had granular cell tumors, a giant lentiginous nevus and a nevus flammeus. A child with both relapsing congenital syphilis and severe combined immunodeficiency was presented by Dr. Karen Sherwood.

Dr. Lynne Roberts emphasized the use of sodium chloride split skin studies to help distinguish bullous pemphigoid from epidermolysis bullosa acquisita, her patient's disease. An infant with epidermolysis bullosa dystrophica with harlequin features was described by Dr. Liz Beightler.

Two cases were reported by Dr. Lawrence Eichenfeld. The first involved an acrodermatitis enteropathica-like eruption with cystic fibrosis. The second case was a $11 / 2$-year-old with an enlarging tongue mass that was diagnosed as Riga-Fede disease. The child was also found to have familial dysautonomia.

The statistics of sporadic dysplastic nevi as melanoma risk markers in relatives were reviewed by Dr. Lee Albert. Dr. Ronald Hansen discussed the use of cooling suits for hypohidrotic ectodermal dysplasia. A handout listing the address and phone number of the Hypohidrotic Ectodermal Dysplasia Foundation (1-804-826-0065) was provided. Dr. Adelaide Hebert described two siblings with the DeSanctis-Cacchione syndrome. The fibroblast analysis on these patients was performed by Dr. James Cleaver, who will address the Society for Pediatric Dermatology at the pre-American Academy of Dermatology meeting in San Francisco, California in December 1989. 
This document is a scanned copy of a printed document. No warranty is given about the accuracy of the copy. Users should refer to the original published version of the material. 